\title{
On the phenomenon of impact
}

\author{
Stephan Kalapis ${ }^{1 *}$, Athanasios Dafnis ${ }^{1}$, and Kai-Uwe Schröder ${ }^{1}$ \\ ${ }^{1}$ Institute of Structural Mechanics and Lightweight Design of RWTH Aachen, Wüllnerstraße 752062 \\ Aachen, Germany
}

\begin{abstract}
Aerostructures such as plates are commonly used in aircraft structures due to their advantageous weight to stiffness ratio. Plates are susceptible to lateral forces that occur during impact. Such impact events are caused by falling tools, bird strikes, collisions of objects, and more. Several researchers have studied impact events, but not yet the behaviour of the plate and impactor shortly after impact due to various factors. In this paper, the semi-analytical approach of Karas is used to calculate key parameters for the analysis of an impact. Karas derived this approach to calculate an impact for a simply supported plate that is impacted in the center. Here, this approach is extended to calculate the impact event at any location on the plate. The linear semi-analytical approach is written in a Python script to obtain fast results. In a code-to-code analysis, this Python code is verified with a commercial FE solver. Various parameters are varied and investigated to analyze and describe the impact phenomenon. In addition to the influence of the initial velocity of the impactor, the mass ratio of the two bodies and the impact location are also investigated. The analysis shows that the contact force and maximum displacement during an impact varies depending on the impact location, while all other parameters are kept constant. Depending on the impact location, the contact force between impactor and plate can be nine times higher than the lowest value.
\end{abstract}

\section{Introduction}

Damage due to impact events are a threat to CFRP and metal plates. The hereby induced damage is often not noticed or is not visible with bare eyes. These damages may lead to failure of the overall structure. To prevent such failures, the impact event must be completely understood, particularly which variables have the greatest influence. With this better understanding of plate behaviour, the prediction of damage before it occurs is possible.

There has been extensive research in the area of low velocity impact. Although such impact events are highly dynamic, Karas was able in 1939 to present an analytical solution to determine contact force and deflection of the plate for a simply supported isotropic plate while it is hit at the center [1]. This approach neglects the effect of membrane and shear forces. Karas' solution can be considered as exact as long as the outer boundary condition meet the physical criteria which are assembled in his theory. Thus, his work is used by other researchers to validate their own work [2-3].

Further detailed research activities on impact events were conducted by Olsson. He distinguished the impact into small and large mass impact events [4]. While small mass

* Corresponding author: stephan.kalapis@sla.rwth-aachen.de 
impacts tend to have peak impact forces with trailing plate deflections, the impact force and plate deflection of large mass impacts are in phase. This can be seen in Figure 1.

Small Mass Impact

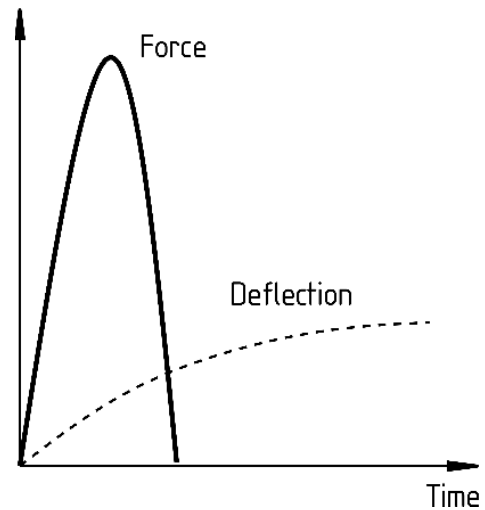

Large Mass Impact

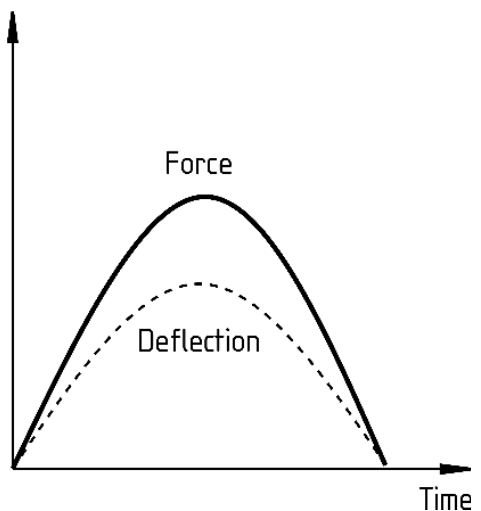

Fig. 1. Schematic differentiation between small and large mass impact.

The small mass impact occurs if the impactors mass is lighter than 0.29 times the plates mass, and a large mass impact occurs if the impactors mass exaggerates the plates mass twice. Thus it is worth noting, that two impacts with the same kinetic energy but different masses and velocities may lead to different damages in a plate.

Within this paper, the initiated damage in a plate is considered to be dependent directly by the contact force. Thus, higher contact forces between impactor and plate leads to bigger damaged areas and lower contact forces to smaller damaged areas, respectively.

\section{Methodology}

In order to calculate the impact on arbitrary position analytically, the work of Karas is chosen to be further investigated. With the analytical solution it is possible to calculate the dynamic deflection of impactor and plate during an impact event as well as the corresponding contact force between them.

As seen in figure 2, the plate is considered to have the width $a$, length $b$ and depth $h$. The impactor has the mass $m_{g}$ and radius $r$, while hitting the plate with the velocity $v_{0}$. Plate and impactor are made out of the same isotropic material with Youngs Modulus E, Poisson's Ratio $v$ and density $\rho$. As the impact location is considered to be arbitrary, this location is described by $\xi$ and $\eta$. 


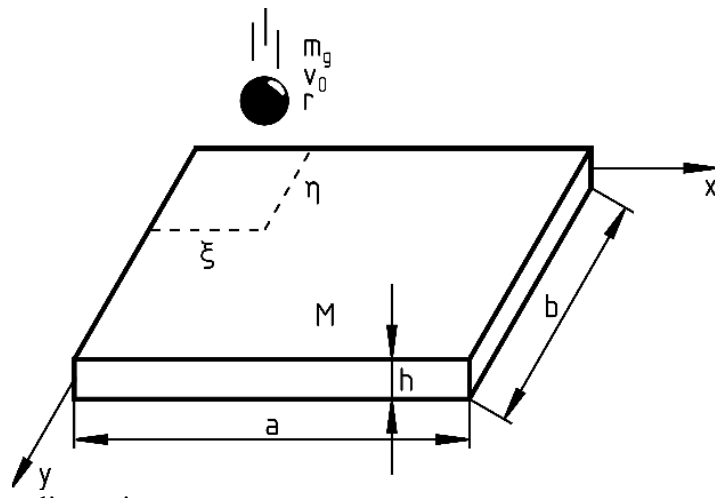

Fig. 2. Plate and impactor dimension.

Karas derived the following equation, which is based on the Kirchhoff hypothesis, to calculate the plate deflection $\mathrm{w}[1]$ :

$$
\begin{aligned}
w(x, y)= & \sum_{m} \sum_{n} \frac{1}{\pi^{2} \bar{a}\left(\frac{m^{2}}{n^{2}}+\frac{n^{2}}{b^{2}}\right)} \frac{4}{\rho h a b} \int_{0}^{t} Q_{m n}(\tau) \sin \left[\left(\frac{m^{2}}{a^{2}}+\frac{n^{2}}{b^{2}}\right) \pi^{2} \bar{a}(t-\tau)\right] d \tau \\
& \times \sin \left(\frac{m \pi x}{a}\right) \sin \left(\frac{n \pi y}{b}\right)
\end{aligned}
$$

with

$$
\bar{a}=\sqrt{\frac{E h^{2}}{12 \rho\left(1-v^{2}\right)}}
$$

Where $m, n$ are even numbers and $t$ the considered time. The expression $Q_{m n}$ is the generalized force. In his work, Karas set the generalized force to be the contact force $P$. This expression is here developed to be

$$
Q_{m n}(\tau)=P(\tau) \sin \left(\frac{m \pi \xi}{a}\right) \sin \left(\frac{n \pi \eta}{b}\right)
$$

In contrast to Karas, who focused on an analytical formulation, this variant is concerned with the arbitrariness of the impact location. As mentioned above, this paper is not about the formulation of a new analytical approach, but about the effects of different impact locations.

As $P$ and $w$ in Eq. 3 are unknown, another equation is needed to substitute the deflection $w$. As the impactor intendates the plate, a Hertzian Contact is obtained. This indentation and the plate deflection $w$ must yield the overall travelled distance $u$ of the impactor. While the indentation is calculable by the Hertzian Contact law, the position of the impactor is determined by following equation:

$$
v_{0} t-\frac{1}{m} \int_{o}^{t} P(\tau)(t-\tau) d \tau
$$

Together with Eq. 3, Eq. 4 and the contact law, one is able to calculate the impact force $P$ by numeric integration. To determine the plates deflection, Eq. 1 must be solved. Here, this routine is automated via a Python-Script. Within this script, it is possible to change the evaluation location $x, y$ independently from the impact location $\xi$ and $\eta$.

This approach considers only linear bending of the plate as Kirchhoff hypothesis for bending plates is included, while non-linear effects such as membrane or shear forces are neglected. 


\section{Numerical verification}

To verify the derived equation, the obtained results are compared with a numeric finite element analysis (FEA). The program used is Abaqus, Dassault Systems. A plate with the dimensions of $200 \times 200 \times 8 \mathrm{~mm}^{3}$ is impacted by a sphere with radius $10 \mathrm{~mm}$ and an initial velocity of $1000 \mathrm{~mm} / \mathrm{s}$. Both plate and impactor are made out of steel with Youngs Modulus of $251,820 \mathrm{~N} / \mathrm{mm}^{2}$ and Poissons Ratio of 0,3. As seen in figure 3, three different locations are evaluated: mid, edge and corner. Following figure 2, the coordinates for the impacts are $(100 \mathrm{~mm} \mid 100 \mathrm{~mm})$ for mid, $(50 \mathrm{~mm} \mid 100 \mathrm{~mm})$ for edge and $(50 \mathrm{~mm} \mid 50 \mathrm{~mm})$ for corner. The simply supported plate consists of 1,156,000 C3D8R elements, the impactor consists of $107,200 \mathrm{C} 3 \mathrm{D} 8 \mathrm{R}$ elements. While the plate deflection can be measured in the post-processing, the impact force must be calculated separately. Therefore, the average acceleration of the impactor is extracted and multiplied by its mass. Using Newtons Second Law the impact force is now obtained.

a)

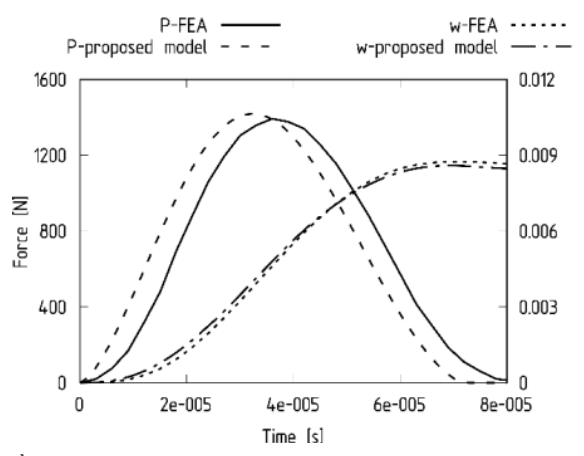

c)

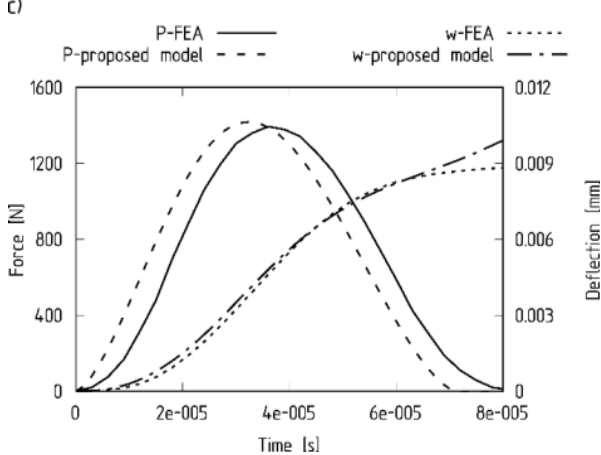

)

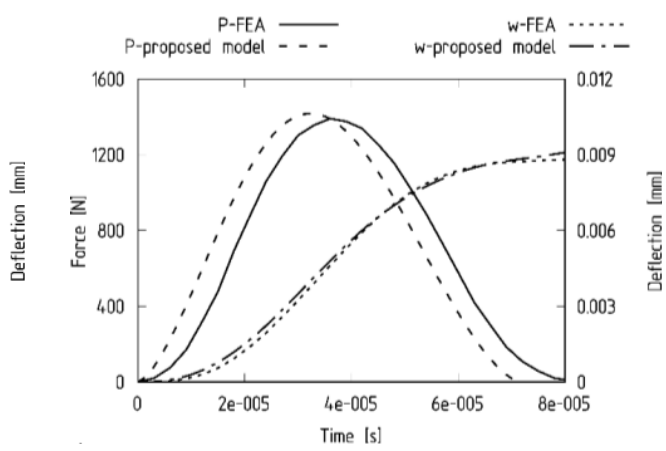

Fig. 3. Verification of the proposed model with FEA. Impact occurs a) in the mid, b) in the side and c) in the corner of the plate.

Following figure 3, the calculated plate deflection and the contact force between plate and impactor of the Python-Script matches the FEA well, regardless the impact location. Hence, there are some minor differences between proposed model and FEA: a slight shift for the impact force and diverging deflections of the plate to the end of the impact event. This circumstance can be justified by the non-linear calculation of the FEA, while the proposed model uses linear approaches. Similar differences were reported by other authors as well [5$6]$.

Furthermore, it was verified that the results are perfectly symmetric along $\mathrm{x}$ - or $\mathrm{y}$ - axis or the diagonal thereof. Thus, the numerical Python-Script is found to work correctly. 


\section{Analytical Investigation and Discussion}

As discussed in section 1, it is important to distinguish between a small mass and a large mass impact. They may lead to different contact forces and thus to different damage-affected areas. Moreover, it is worth knowing if and how these contact forces differ on arbitrary plate location.

For this purpose, a small mass and a large mass impact are studied on arbitrary impact locations. For both types of impact the kinetic energy equals $10 \mathrm{~J}$. The initial velocity is set to fulfil the given mass and level of kinetic energy. The investigated plate is the same as in section 3, thus it has a dimension of $200 \times 200 \times 8 \mathrm{~mm}^{3}$ and is made out of steel with Young's Modulus of $251,820 \mathrm{~N} / \mathrm{mm}^{2}$ and Poissons Ratio of 0,3. An isotropic material is choosed as Eq. 1 can only handle such materials. For investigations on anisotropic materials such as CFRPs, a quasi-isotropic laminate must be chosen. In figure 4 the maximum deflection of the plate, the maximum contact force and the number of subsequent impacts in such an event is shown for both types of impact. The calculated time was chosen to guarantee that no contact between plate and impactor is missed.

small mass impact, $M R=0.2, E_{\text {kin }}=10 \mathrm{~J}, v_{0}=6.27 \mathrm{~m} / \mathrm{s}$
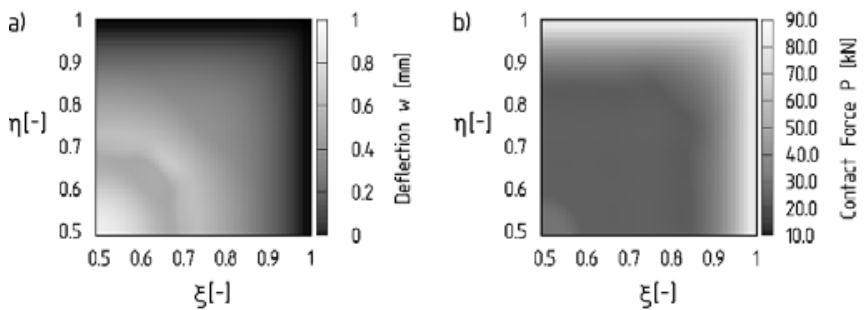

large mass impact, $M R=1.0, E_{\text {kin }}=10 \mathrm{~J}, v_{0}=2.8 \mathrm{~m} / \mathrm{s}$
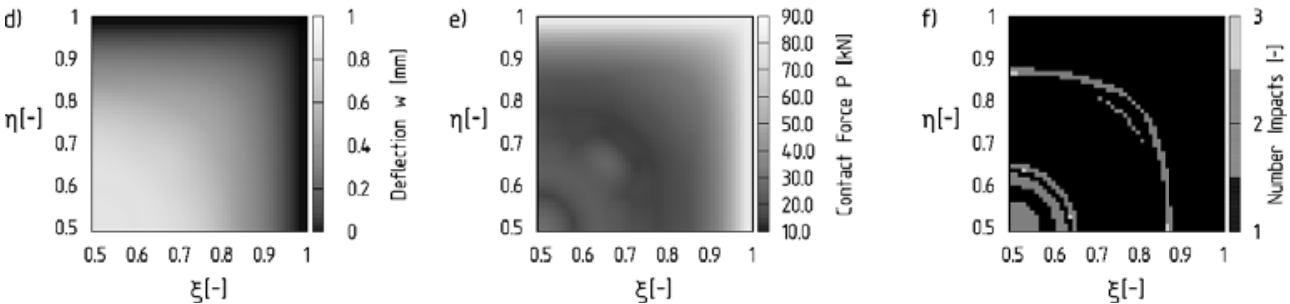

Fig. 4. Evaluation of plate deflection, contact force and number of impacts for small and large mass impact on arbitrary position.

On the first look it is evident, that the deflection and force distribution over the plate are not equal for large and small mass impact. Although, as expected, the deflection decreases approaching the boundary while the contact force increases. This effect leads back to the higher plate stiffness near the boundary and no much movement of the plate is observed. This explanation is backed by the fact, that in the boundary region only one contact during the impact event occurs.

For the small mass impact, more dynamic events are observed in the middle of the plate. Here, two to four single contacts are occurring leading to diverging maximum deflection of the plate. Interestingly, the maximum contact force is not much affected in the middle of the plate: from the center to the boundary the contact forces decreases a little bit and then it raises to the boundary.

The large mass impact behaves the opposite way than the small mass impact. Here are mainly one, at some regions in circles around the center two, contacts in the impact event. While the maximum deflection reduces from the center to the boundary as expected, the 
contact force has some local abnormalities. Around the center exists a circle, where the contact force is dropping. Around the area at $(0.68 \mid 0.68)$ a local maximum is observed.

Figure 4 shows the high dependency of the location on impact events regarding the plates deflection and contact force. If the impact event happens just $10 \mathrm{~mm}$ further away, a different damage area could be observed. Although the derived equations are only valid for thin plates and the here investigated plate does not fit that criteria well, the here obtained results can be used for a first rough estimation. The influence of shear or membrane forces is considered to be negligible in this case.

\section{Conclusion}

In this paper the impact event of a round sphere on a flat plate is investigated. The analytical approach of Karas was expanded to calculate impact events on arbitrary plate positions by using a more detailed approach for the generalized force. The here analytical derived approach was compared and verified with a commercial FEA.

Here, it was shown that the impact location has a tremendous influence on the impact event, regarding its plate deflection and contact force history. Thus, it appears that some areas on a plate are more susceptible to damages by impacts. These areas must not necessarily lay in the middle of the plate but a little bit outside of it. Furthermore, the mass ratio influences the position of this area. A general pattern, where the maximum contact force can be expected is not given here, though it must be further researched.

Focus in further research must be given on the detailed dynamics of the impact and the here involved parts. The driving variables must be identified, which will lead to the differentiation of small and large mass impact.

The authors declare that they have no known competing financial interests or personal relationships that could have appeared to influence the work reported in this paper.

\section{References}

[1] K. Karas, Ing.-Arch. (10) (1939) 237-250. doi:10.1007/BF02084907

[2] S. Khalili, K. Malekzadeh, A. V. Gorgabad, Compos. Struct. 96 (2013) 64-74. doi:10.1016/j.compstruct.2012.07.035.

[3] D. Chakraborty, Mater. Des. 28 (4) (2007) 1142-1153. doi:10.1016/j.matdes.2006.01.029.

[4] R. Olsson, Compos. Part A Appl. Sci. Manuf. 31 (8) 490 (2000) 879-887. doi:10.1016/S1359-835X(00)00020-8.

[5] M. R. Bayat, O. Rahmani, M. Mosavi Mashhadi, Polym. Compos. 39 (3) (2018) 730745. doi:10.1002/pc.23990.

[6] M. Ghalami-Choobar, G. Liaghat, M. Sadighi, H. Ahmadi, Compos. Struct. 201 (2018) 315-325. doi:10.1016/j.compstruct.2018.06.027. 